\title{
On the development of a 3D cohesive zone element in the presence of large deformations
}

\author{
M. J. van den Bosch • P. J. G. Schreurs • \\ M. G. D. Geers
}

Received: 13 October 2006 / Accepted: 11 April 2007 / Published online: 15 May 2007

(C) Springer-Verlag 2007

\begin{abstract}
Delamination is typically modelled using cohesive zone models. In this paper, it is shown that the validity of most models is limited to small displacements and/or deformations at the interface and the surrounding bulk materials. A large displacement formulation (LDF) is proposed that overcomes issues of the classical formulation with large displacements and deformations at the interface. Subsequently, a 3D cohesive zone element with this LDF is introduced and its numerical implementation is elaborated. Then, a 3D FEM model is proposed and the determination of several model parameters is substantiated. Finally, the implementation is validated by comparing numerical results with experimental observations.
\end{abstract}

Keywords Cohesive zone - Large displacements . Delamination $\cdot$ Laminates $\cdot$ Coatings

\section{Introduction}

Polymer coated metals are used nowadays for an extending variety of products, e.g., beverage cans and aerosols. A polymer coating is applied to the metallic substrate prior to forming a product. Polymer coated metals have several advantages over uncoated metals. The polymer coating protects the substrate from corrosion, without influencing the taste of the beverage or food. Moreover, costs savings are

M. J. van den Bosch

Netherlands Institute for Metals Research (NIMR),

PO Box 5008, 2600 GA Delft, The Netherlands

M. J. van den Bosch • P. J. G. Schreurs · M. G. D. Geers ( $\square)$

Department of Mechanical Engineering,

Eindhoven University of Technology, PO Box 513,

5600 MB Eindhoven, The Netherlands

e-mail: m.g.d.geers@tue.nl possible because there are no emissions of volatile organic compounds and lacquering is superfluous $[5,10]$.

The polymer coating can delaminate because it is subjected to the same deformation as the metal substrate. This causes the loss of protective and attractive properties of the product [3] and is therefore unacceptable. Adequate material description and process simulations are required to predict delamination, which allows the adjustment of process parameters to prevent it.

In-situ observation of the delamination process of polymer coated steel reveals the presence of large deformations and displacements in both the interface and the bulk materials. The delamination occurs by the initiation, growth and fracture of fibrils at the interface, a process called fibrillation.

In the numerical tool, the delamination process is described by cohesive zone models, which represent a relation between the traction and the relative displacement of associated points of crack surfaces. Cohesive zones are typically implemented in finite element codes as interface elements.

Most (2D) cohesive zone models provide a constitutive relation between the normal opening displacement and the normal traction and a separate relation between the tangential opening displacement and the tangential traction (see a.o. $[4,7,18]$ for an overview). The tractions are sometimes coupled by making them a function of both the normal and tangential opening displacement [17,21]. The normal and tangential direction are defined with respect to a local coordinate basis that is placed inside the cohesive zone element. This approach is accurate when there are small displacements present at the interface, e.g., when the cohesive zones are used to simulate the indentation-induced delamination of a brittle film [1]. Therefore these models are next referred to as cohesive zone models with a small displacement formulation (SDF). However, in the presence of large displacements at 
the interface, these models, as shown later, may give unexpected results. To overcome the problems of the SDF, a large displacement formulation (LDF) for fibrillation is proposed.

Large displacement cohesive zone models have already been developed $[14,15]$. However, these cohesive zone models were used to predict crack growth with large displacements in the bulk material but the displacements at the interface prior to failure were relatively small and should therefore be considered as SDF models.

In this paper first some experimental results on the delamination of the polymer coating are shown, illustrating the formation of fibrils. Secondly, the SDF and its problems to describe large displacements unambiguously are discussed. Subsequently, the LDF is introduced and it is shown how it handles large displacements correctly. Then, the formulation of a 3D cohesive zone element with a LDF is elaborated. Finally, the constitutive model for the coating is introduced, on the basis of which a 3D peel model is built and compared with experiments.

\section{Experimental observation of fibrillation}

The substrate is a batch annealed steel. It is extrusion coated on one side with poly-ethylene terephthalate (PET). The substrate is $210 \mu \mathrm{m}$ thick and the PET layer has a thickness of $30 \mu \mathrm{m}$.

Peel test were performed in-situ inside a scanning electron microscope (SEM) as shown in Fig. 1 [19]. At the delamination front, the formation, growth and debonding of fibrils was observed (see Fig. 2). This process is called fibrillation and is considered to be the main mechanism by which the coating delaminates from the substrate under the imposed loading conditions.

\section{Small displacement formulation}

Most cohesive zone models in literature use a SDF to describe quasi-brittle fracture. These models may have two or three

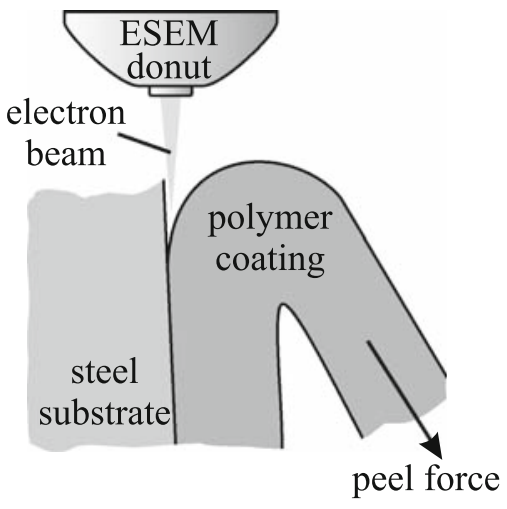

Fig. 1 In-situ delamination setup

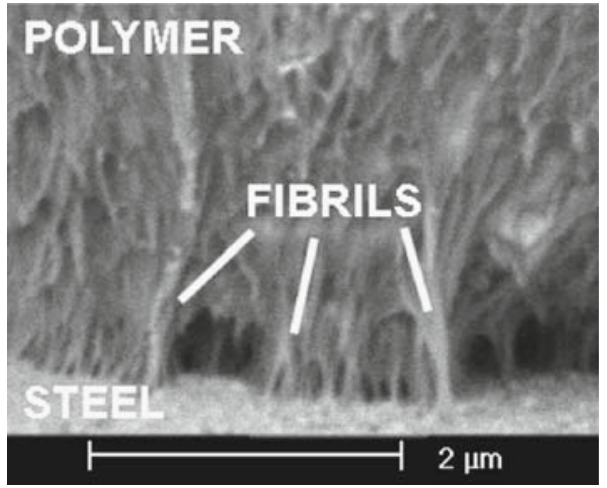

Fig. 2 SEM micrograph of the delamination front and the presence of fibrils

constitutive relations $(2 \mathrm{D} / 3 \mathrm{D})$, that describe the relations between the tractions and the opening displacements. Here a reversible exponential cohesive zone law is used [18]:

$T_{t}=2 \frac{\phi_{t}}{\delta_{t}}\left(\frac{\Delta_{t}}{\delta_{t}}\right)\left(1+\frac{\Delta_{n}}{\delta_{n}}\right) \exp \left(-\frac{\Delta_{n}}{\delta_{n}}\right) \exp \left(-\frac{\Delta_{t}^{2}}{\delta_{t}^{2}}\right)$

and

$T_{n}=\frac{\phi_{n}}{\delta_{n}}\left(\frac{\Delta_{n}}{\delta_{n}}\right) \exp \left(-\frac{\Delta_{n}}{\delta_{n}}\right) \exp \left(-\frac{\Delta_{t}^{2}}{\delta_{t}^{2}}\right)$

where $T$ represents a traction, $\phi$ a work-of-separation, $\delta$ a characteristic cohesive length and $\Delta$ an opening displacement. The subscripts ()$_{t}$ and ()$_{n}$ refer to the tangential and normal directions with respect to a local basis.

Figure 3 shows a quadrilateral cohesive zone element with four nodal points and two integration points. The opening displacements are determined by decomposing the total opening displacement with respect to a local orthonormal basis $\left\{\mathbf{e}_{t}, \mathbf{e}_{n}\right\}$. The local basis is commonly defined with respect to a reference line. In most cases this is the cohesive zone mid-line, which is line A-B in Fig. 3.

If there are small displacements at the interface, all reference lines within a cohesive zone element are parallel to each other, i.e., the choice for a specific reference line will
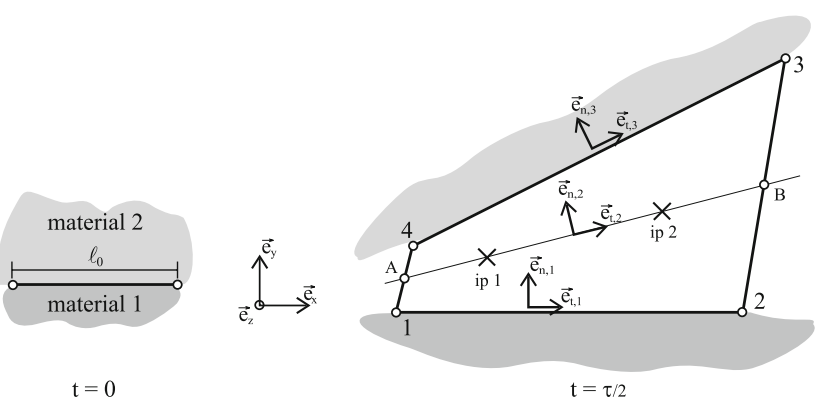

Fig. 3 Schematical representation of the initial and deformed geometry of a planar cohesive zone element with large displacements and three possible orientations of a local basis 


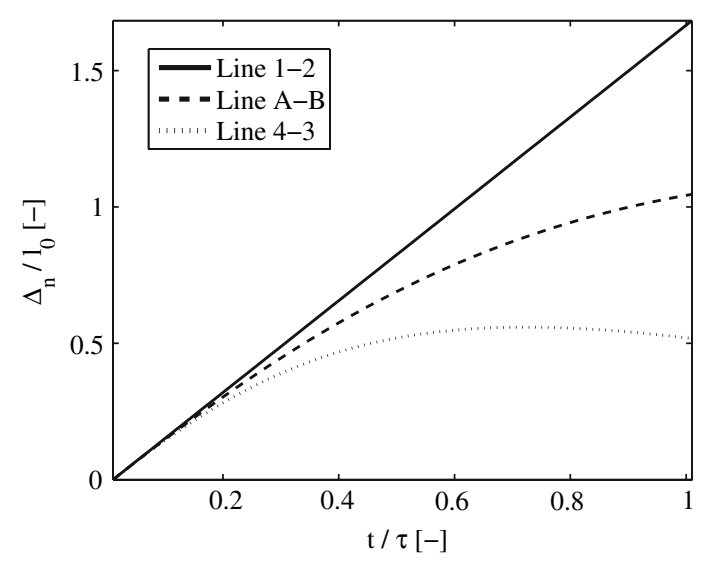

Fig. 4 The calculated normal opening displacement (normalized by the initial cohesive zone length $\ell_{0}$ ) of integration point 1 (ip 1 in Fig. 3) for three different local bases as a function of the time $t$ normalized by time $\tau$ at which the maximum opening is achieved

not significantly influence the magnitude of the normal and tangential opening displacement. However, when large displacements are present at the interface, the choice of a reference line may influence the orientation of the local basis and hence the decomposition of the total opening displacement in a normal and tangential contribution [6]. To illustrate this, a single cohesive zone element is monotonically deformed in a typical deformation mode shown in Fig. 3. The normal opening displacements, calculated with respect to the three local bases, are shown in Fig. 4. From Fig. 4 it is clear that the normal opening displacement cannot be determined unambiguously due to the differences in orientation of the local bases.

So, the use of a local basis has several consequences when large displacements occur at the interface. First, the orientation of the local basis will influence the magnitude of $\Delta_{t}$ and $\Delta_{n}$ and consequently the contribution of $\phi_{t}$ and $\phi_{n}$ to the total dissipated energy. Secondly, in most SDF implementations the integration of the tractions is performed over the length of the reference line. Upon large displacements in one of the joined materials, the lengths of the reference lines will change during deformation and as a result, the integrated tractions will have different magnitudes.

Since delamination in polymer coated steel is characterized by fibrillation and large displacements at the interface, a solution is required to remedy the inconsistencies that may result from the choice of the local basis.

\section{Large displacement formulation}

If there are large displacements present at the interface, it is no longer physical to uniquely distinguish between a normal and tangential opening. In the particular case of polymer coated steels, the interfacial gap is bridged by fibrils that can

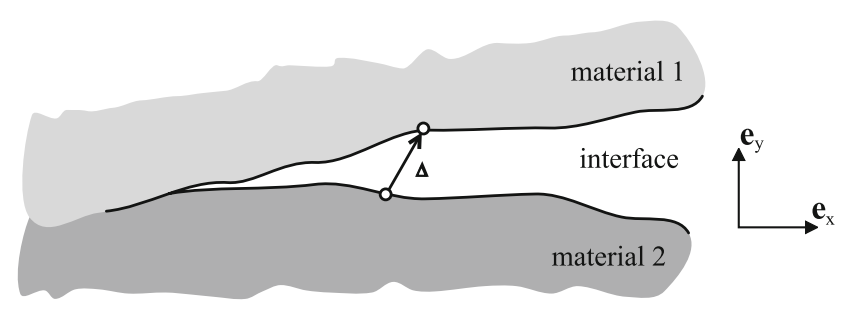

Fig. 5 An interface between two materials with an opening displacement $\boldsymbol{\Delta}$ between two points that coincided initially

only transfer a load along their axes. A LDF is therefore proposed to resolve the ambiguity induced by the choice of a local basis, where no distinction will be made between normal and tangential loadings. Only a single constitutive relation between the traction $\mathbf{T}$ and the opening displacement $\boldsymbol{\Delta}$ is proposed:

$\mathbf{T}=\mathbf{f}(\Delta)$.

The opening displacement is calculated between two points, on both sides of the interface, which coincided initially as sketched in Fig. 5. The vectors are no longer decomposed with respect to a local basis but are resolved globally. Therefore, the deficiencies of the SDF are overcome, since there is no need for a local basis. Adopting the geometrically nonlinear solution procedure proposed in [19], tractions are resolved as Piola-Kirchhoff tractions. In here, the required cohesive zone length is the initial length, which can be determined unambiguously.

The constitutive relation (3) is next explicated on the basis of the normal traction of the SDF cohesive zone law (Eq. 2). It relates the traction $\mathbf{T}=T \mathbf{e}$ to the opening displacement $\boldsymbol{\Delta}=\Delta \mathbf{e}$, where $\mathbf{e}$ is the unit vector along the line between the associated points of the interface:

$T=\frac{\phi}{\delta}\left(\frac{\Delta}{\delta}\right) \exp \left(-\frac{\Delta}{\delta}\right)$

where $\phi$ is the work-of-separation and $\delta$ characteristic opening length, see Fig. 6. The maximum traction $T_{\max }$ is related to $\phi$ and $\delta$ by:

$T_{\max }=\frac{\phi}{\delta \exp (1)}$.

As shown in Fig. 6, upon unloading, the cohesive zone law can show a reversible response (i) or two types of irreversible behaviour: linear elastic unloading to the origin, i.e., elasticity-based damage (ii) and unloading with the initial stiffness of the cohesive zone, i.e., plastically damaged (iii). All three cases are shown in Fig. 6.

It has been shown previously that the shape of the cohesive zone law, even when a large deformation formulation is used, has no significant influence on the macroscopic response of the model [19]. In this paper, an exponential cohesive zone law is used because its traction and derivative are continuous 


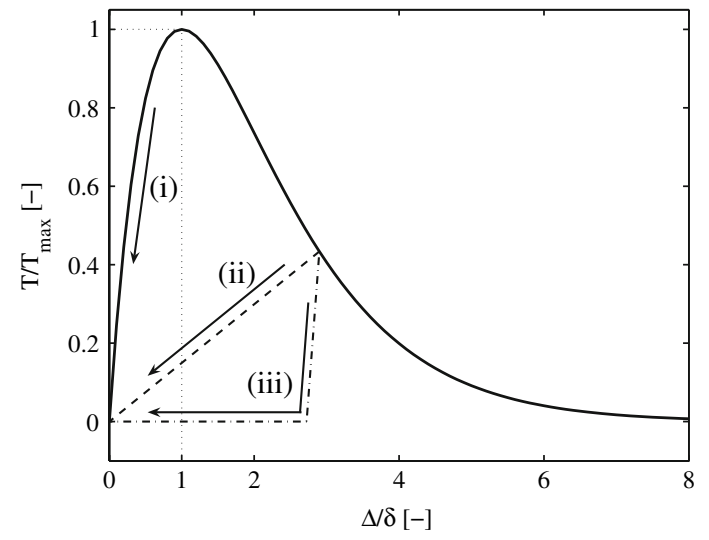

Fig. 6 Cohesive zone law of equation (4) with the maximum traction $\Delta=\delta$ and three types of unloading behaviour

in forward loading, which is attractive from a computational point of view.

There are a number of advantages of a LDF cohesive zone model compared to a SDF one. Firstly, there is no local basis, rendering the decomposition of the total displacement superfluous. Therefore, the total work-of-separation is always equal to $\phi$, independent of the mode-mixity. This is a valid simplification of a mode-dependent cohesive zone law if there are only fibrils at the interface. Secondly, the cohesive zone law of equation (4) can be derived from a potential function, which makes the dissipated energy intrinsically path-independent. Finally, the first Piola-Kirchhoff tractions are integrated in a consistent unambiguous manner by using the initial cohesive zone element length.

\section{Three-dimensional cohesive zone element}

The cohesive zone is implemented in the commercial finite element code MSC.MARC as a user element in a large displacement framework. The weak form of the weighted residual integral of the equilibrium equation reads:

$$
\int_{V}(\nabla \mathbf{w})^{c}: \mathbf{S} d V=\int_{V} \mathbf{w} \cdot \mathbf{q} d V+\int_{A} \mathbf{w} \cdot \mathbf{t} d A \quad \forall \mathbf{w}(\mathbf{x})
$$

where $\nabla$ is the gradient operator and $\mathbf{w}$ arbitrary continuous weighting function. The boundary tractions at the external boundary $A$ are given by $\mathbf{t}=\mathbf{S} \cdot \mathbf{n}$, whereas the body forces q apply to the volume $V$. The sum of the two integrals with the external load vectors are denoted as the external force integral $f_{e}$. The remaining integral on the left hand side is the internal force integral $f_{i}$, which expresses the virtual work of the internal stresses and the cohesive zone tractions. The weighted residual integral equation is then compactly written as:

$f_{i}(\mathbf{w}, \mathbf{S})=f_{e}(\mathbf{w}, \mathbf{t}, \mathbf{q}) \quad \forall \mathbf{w}$.
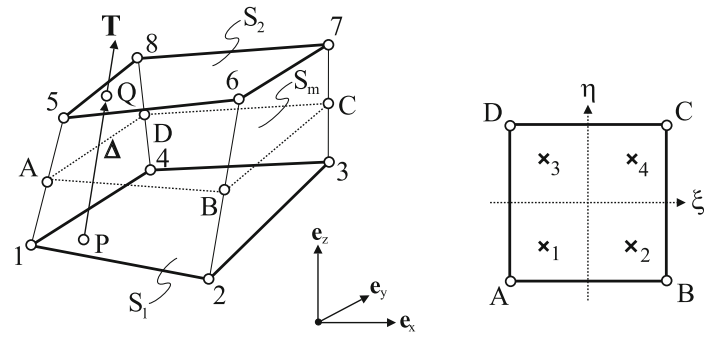

Fig. 7 An eight-noded 3D cohesive zone element with four integration points

A 3D cohesive zone element is shown in Fig. 7. Both the local coordinates, $\xi$ and $\eta$, span the range [ -11$]$. In the deformed state a traction $\mathbf{T}$ connects associated (= with the same $(\xi, \eta)$ coordinate) points $\mathrm{P}$ and Q located on the faces $S_{1}$ and $S_{2}$, respectively. Focussing attention to the cohesive zone element, traction equilibrium across the interface involves the (Cauchy) traction vector $\sigma$ rather than the full stress tensor $\mathrm{S}$. Integration over the cohesive zone element volume with area $A$ yields:

$f_{i}=\int_{A} \Delta \mathbf{w} \cdot \boldsymbol{\sigma} d A$

where the weighting function can be considered as a kinematically admissible virtual displacement. A pull-back to the initial undeformed state is performed, where the integration takes place over the initial area $A_{0}$. Accordingly, Eq. (8) becomes:

$f_{i}=\int_{A_{0}} \Delta \mathbf{w} \cdot \mathbf{T} d A_{0}$

where $\mathbf{T}$ is the first Piola-Kirchhoff traction vector. The weighting function $\mathbf{w}$ and the traction $\mathbf{T}$ are a function of the local coordinate $\eta$. The integration is expressed in this coordinate, leading to:

$\underset{\sim}{f_{i}}=\frac{A_{0}}{4} \int_{-1}^{1} \int_{-1}^{1} \underline{P}^{T} \underline{N}^{T}(\xi, \eta) \underset{\sim}{T}(\xi, \eta) d \xi d \eta$

where $\underset{\sim}{T}(\xi, \eta)$ is the column with the tractions and $\underline{P}$ is an operator matrix that transforms the nodal quantities to the points $\mathrm{A}, \mathrm{B}, \mathrm{C}$ and $\mathrm{D}$ :

$\underline{P}=\left[\begin{array}{ll}-\underline{I}_{12} & \underline{I}_{12}\end{array}\right]$

in which $\underline{I}_{12}$ is a $12 \times 12$ unity matrix. $N(\xi, \eta)$ is the matrix with the interpolation functions:

$\underline{N}(\xi, \eta)=\left[\begin{array}{llllllllllll}\psi_{1} & 0 & 0 & \psi_{2} & 0 & 0 & \psi_{3} & 0 & 0 & \psi_{4} & 0 & 0 \\ 0 & \psi_{1} & 0 & 0 & \psi_{2} & 0 & 0 & \psi_{3} & 0 & 0 & \psi_{4} & 0 \\ 0 & 0 & \psi_{1} & 0 & 0 & \psi_{2} & 0 & 0 & \psi_{3} & 0 & 0 & \psi_{4}\end{array}\right]$ 
in which $\psi_{i}$ are the four shape functions associated with the points $\mathrm{A}, \mathrm{B}, \mathrm{C}$ and $\mathrm{D}$ :

$$
\begin{array}{ll}
\psi_{1}=\frac{1}{4}(1-\xi)(1-\eta) & \psi_{2}=\frac{1}{4}(1+\xi)(1-\eta) \\
\psi_{3}=\frac{1}{4}(1+\xi)(1+\eta) & \psi_{4}=\frac{1}{4}(1-\xi)(1+\eta) .
\end{array}
$$

Following a standard iterative procedure, similar to the classical 2D implementations, yields the approximate incremental value of the internal load integral and the element stiffness matrix:

${\underset{\sim}{f}}_{i}^{*}=\frac{A_{0}}{4} \sum_{\mathrm{ip}=1}^{4}\left[\underline{P}^{T} \underline{N}^{T}(\xi, \eta) \underset{\sim}{T}(\xi, \eta)\right]^{\mathrm{ip}}$

and

$\underline{K}^{*}=\frac{A_{0}}{4} \sum_{\mathrm{ip}=1}^{4}\left[\underline{P}^{T} \underline{N}^{T}(\xi, \eta) \underline{M}(\xi, \eta) \underline{N}(\xi, \eta) \underline{P}\right]^{\mathrm{ip}}$

where ( $)^{*}$ denotes the incremental value and $\underline{M}$ is the cohesive tangent operator. Finally, the iterative element equation reads:

$\underline{K}^{*} \delta \underset{\sim}{u}=f_{\sim e}-f_{\sim i}^{*}$

where $\delta \underset{\sim}{u}$ is a column with the iterative nodal displacements.

\subsection{Contact}

A contact algorithm is required to detect the penetration of surface $S_{1}$ into $S_{2}$, or vice versa. The opening displacement in the LDF is always positive $(\Delta=\|\boldsymbol{\Delta}\|)$ and can therefore not be used to detect penetration. The approach presented here firstly determines a normal vector $\mathbf{n}$ to the middle surface $S_{m}$ of the cohesive zone. Then, it decomposes $\boldsymbol{\Delta}$ to find the component parallel to $\mathbf{n}$. If this component is negative, there is penetration and a contact penalization is invoked.

This approach apparently introduces a local basis into the formulation. However, this only affects the solution in the case of contact without significant influences on the solution in relation to other local bases.

Since the surface $S_{m}$ has four nodes, it can be curved and there is no consistent normal vector. The normal vector is determined by taking the cross product of two tangent vectors. The two tangent vectors, $\mathbf{t}_{\xi}$ and $\mathbf{t}_{\eta}$, are determined with respect to the isoparametric coordinate directions in the isoparametric mid-point $M(\xi=0, \eta=0)$, as shown in Fig. 8 . The tangent vectors are given by:

$$
\mathbf{t}_{\xi}=\left.\frac{\partial \mathbf{x}}{\partial \xi}\right|_{M} \quad \text { and } \quad \mathbf{t}_{\eta}=\left.\frac{\partial \mathbf{x}}{\partial \eta}\right|_{M}
$$
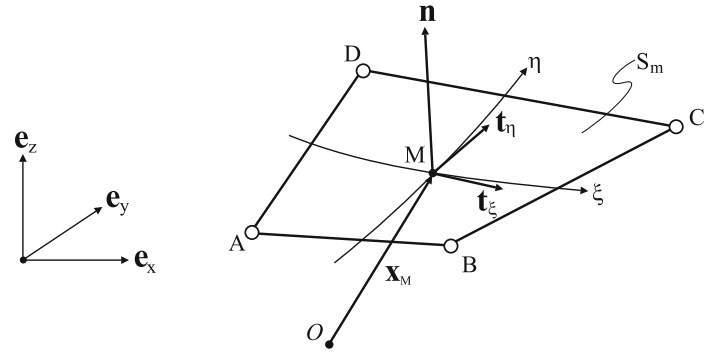

Fig. 8 The normal vector $\mathbf{n}$ is determined in point $\mathbf{M}$ by the cross product of two tangent vectors

The normal vector is given by the normalized cross product of the two tangent vectors:

$\mathbf{n}=\frac{\mathbf{t}_{\xi} \times \mathbf{t}_{\eta}}{\left\|\mathbf{t}_{\xi} \times \mathbf{t}_{\eta}\right\|}$

The magnitude of penetration parallel to the surface normal $\mathbf{n}$ is $\Delta_{c}=\boldsymbol{\Delta} \cdot \mathbf{n}$, which leads to a contact penetration vector:

$\boldsymbol{\Delta}_{c}=\Delta_{c} \mathbf{n}=(\boldsymbol{\Delta} \cdot \mathbf{n}) \mathbf{n}$.

\subsection{Tractions}

The adopted constitutive relation that relates the opening vector $\Delta$ to its corresponding traction is given by the exponential cohesive zone law [19]:

$T=\frac{\phi}{\delta} \frac{\Delta}{\delta} \exp \left(-\frac{\Delta}{\delta}\right)$

where $\Delta=\|\boldsymbol{\Delta}\|$ and $\phi$ and $\delta$ are the cohesive zone parameters. The traction vector becomes:

$\mathbf{T}=T \mathbf{m}$

where $\mathbf{m}$ is a unit vector directed along $\Delta$. In the case contact is present, i.e., $\Delta_{c}<0$, an extra penetration term $T_{c}$ is present, directed along the normal of the plane:

$T_{c}=-k_{c}\left(\frac{\Delta_{c}}{\delta}\right)^{2} \quad$ and $\quad \mathbf{T}_{c}=T_{c} \mathbf{n}$

where $k_{c}$ is the contact stiffness. The contact penetration term is added to the constitutive traction resulting from the physical opening displacement $\boldsymbol{\Delta}$. This yields the total traction vector:

$\mathbf{T}=\mathbf{T}+\mathbf{T}_{c}$.

\subsection{Tangent operator}

The consistent tangent stiffness tensor M relates infinitesimal variations of the traction vector to infinitesimal variations of the opening displacement vector:

$\delta \mathbf{T}=\mathbf{M} \cdot \delta \boldsymbol{\Delta}$. 
With respect to a Cartesian basis, $\left\{\mathbf{e}_{x}, \mathbf{e}_{y}, \mathbf{e}_{z}\right\}$, Eq. 24 becomes:

$\delta \underset{\sim}{T}=\underline{M} \delta \underset{\sim}{\Delta}$

where components are stored in columns $\delta \underset{\sim}{T}$ and $\delta \underset{\sim}{\underset{\sim}{\Delta} \text { and }}$ matrix $\underline{M}$. The consistent tangent stiffness matrix $\underline{M}$ for the LDF follows from a standard analysis of a 3D truss element under large displacements and rotations:

$\underline{M}=\left(\frac{\partial T}{\partial \Delta}\right)^{*} \underset{\sim}{m} m^{T}+\frac{T^{*}}{\Delta^{*}} m_{2} \underline{\sim}_{2}^{T}+\frac{T^{*}}{\Delta^{*}} \underline{\sim}_{3} \underline{\sim}_{3}^{T}$

where ${\underset{\sim}{\sim}}_{\sim} \underline{\sim}_{2}$ and $m_{\sim}$ are columns containing the components of the vectors $\mathbf{m}, \mathbf{m}_{2}$ and $\mathbf{m}_{3}$ that constitute an orthonormal vector basis. For the exponential cohesive zone law, with characteristic length $\delta$, it can be verified that:

$\frac{T^{*}}{\Delta^{*}}=\frac{\delta}{\delta-\Delta^{*}}\left(\frac{\partial T}{\partial \Delta}\right)^{*}$

which leads to:

$$
\begin{aligned}
\underline{M}=\left(\frac{\partial T}{\partial \Delta}\right)^{*} & \frac{1}{\Delta^{*}\left(\delta-\Delta^{*}\right)} \\
& {\left[\begin{array}{lll}
\delta \Delta^{*}-\Delta_{x}^{2} & -\Delta_{x} \Delta_{y} & -\Delta_{x} \Delta_{z} \\
-\Delta_{x} \Delta_{y} & \delta \Delta^{*}-\Delta_{y}^{2} & -\Delta_{y} \Delta_{z} \\
-\Delta_{x} \Delta_{z} & -\Delta_{y} \Delta_{z} & \delta \Delta^{*}-\Delta_{z}^{2}
\end{array}\right] }
\end{aligned}
$$

where $\Delta_{i}=\boldsymbol{\Delta}^{*} \cdot \mathbf{e}_{i}$, with $i$ the global $x, y$ and $z$ directions. The tangent operator for the contact penalization equals:

$\underline{M}_{c}=\left(\frac{\partial T_{c}}{\partial \Delta_{c}}\right)^{*}\left(\underset{\sim}{n \sim} n^{T}\right)$.

So, in the case of contact the total tangent operator becomes:

$\underline{M}=\underline{M}+\underline{M}_{c}$

\section{Constitutive modelling}

A 3D constitutive model is used for description of large strain, time dependent mechanical behaviour of the polymer layer. The constitutive model is a compressible generalization of the Leonov model [13], proposed by [2]. In the model a distinction is made between the contribution of secondary interactions between polymer chains, which determine the (visco-) elastic properties at small deformations and plastic flow, and the entangled polymer network, which governs the strain hardening. Accordingly, based on the original work of Haward and Thackray [11], the total Cauchy stress $S$ is decomposed in a driving stress $\mathrm{S}_{s}$ and a hardening stress $\mathrm{S}_{r}$ :

$\mathrm{S}=\mathrm{S}_{s}+\mathrm{S}_{r}$

The hardening is modelled with a neo-Hookean relation [16]:

$\mathrm{S}_{r}=G_{r} \tilde{\mathrm{B}}^{d}$

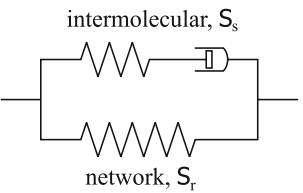

(a)

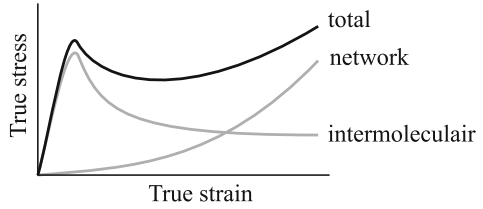

(b)
Fig. 9 a Compressible Leonov model. b Schematic true stress-strain curve of with decomposition of the intrinsic deformation behaviour

where $G_{r}$ is the strain hardening modulus and $\tilde{\mathrm{B}}^{d}$ is the deviatoric part of the isochoric left Cauchy-Green deformation tensor. The driving stress is decomposed into a deviatoric stress $\mathrm{S}_{s}^{d}$ and a hydrostatic stress $\mathrm{S}_{s}^{h}$ :

$\mathrm{S}_{s}^{d}=G \tilde{\mathrm{B}}_{e}^{d}$ and $\mathrm{S}_{s}^{h}=\kappa(J-1) \mathrm{I}$

where $G$ is the shear modulus, $\tilde{\mathrm{B}}_{e}^{d}$ the deviatoric part of the isochoric elastic left Cauchy-Green strain tensor, $\kappa$ the bulk modulus, $J$ the volume change ratio, and I the unity tensor. The evolution of $J$ and $\tilde{\mathrm{B}}_{e}^{d}$ is given by the following kinematical equations:

$\dot{J}=J \operatorname{tr}(\mathrm{D})$

and

$\tilde{\mathrm{B}}_{e}=\left(\mathrm{D}^{d}-\mathrm{D}_{p}\right) \cdot \tilde{\mathrm{B}}_{e}+\tilde{\mathrm{B}}_{e} \cdot\left(\mathrm{D}^{d}-\mathrm{D}_{p}\right)$.

Here $\tilde{\mathrm{B}}_{e}$ is the objective Jaumann rate of $\tilde{\mathrm{B}}_{e}$ and $\mathrm{D}^{d}$ the deviatoric part of the rate of deformation tensor. The plastic deformation rate tensor $D_{p}$ is related to the deviatoric part of the driving stress $\mathbf{S}_{s}^{d}$ :

$\mathrm{D}_{p}=\frac{\mathrm{S}_{s}^{d}}{2 \eta}$.

The viscosity $\eta$ was originally described by an Eyring relationship [8]. It was extended to incorporate pressure dependency and intrinsic strain softening in [9]:

$\eta(T, p, \bar{\tau}, S)=\eta_{0, r}(T) \exp \left(\frac{\mu p}{\tau_{0}}\right) \exp (S) \frac{\bar{\tau} / \tau_{0}}{\sinh \left(\bar{\tau} / \tau_{0}\right)}$

where $\eta_{0, r}$ denotes the zero-viscosity for the completely rejuvenated state, $T$ is the temperature, $\mu$ is the material parameter describing the pressure dependence and $S$ is a parameter that captures the thermal and mechanical history of the material. The hydrostatic pressure $p$, the characteristic stress $\tau_{0}$ and the equivalent stress $\bar{\tau}$ are defined as:

$$
\begin{aligned}
p & =-\frac{1}{3} \operatorname{tr}(\mathrm{S}) \\
\tau_{0} & =\frac{k T}{V^{*}} \\
\bar{\tau} & =\sqrt{\frac{1}{2} \operatorname{tr}\left(\mathrm{S}_{s}^{d} \cdot \mathrm{S}_{s}^{d}\right)}
\end{aligned}
$$


Table 1 PET material parameters

\begin{tabular}{lllll}
\hline$E(\mathrm{GPa})$ & $v(-)$ & $S_{a}(-)$ & $\eta_{0, r}(\mathrm{MPas})$ & $\tau_{0}(\mathrm{MPa})$ \\
\hline 1.25 & 0.4 & 28.4 & 0.486 & 0.95 \\
\hline \hline$\mu(-)$ & $G_{r}(\mathrm{MPa})$ & $r_{0}(-)$ & $r_{1}(-)$ & $r_{2}(-)$ \\
\hline 0.047 & 28.2 & 0.990 & 200 & -4 \\
\hline
\end{tabular}

with $k$ Bolzmann's constant and $V^{*}$ the activation volume. The state parameter $S$ is decomposed into a factor $S_{a}$, which captures the thermo-mechanical history of the material, and $R_{\gamma}\left(\bar{\gamma}_{p}\right)$, which describes the softening kinetics [12]:

$S\left(\bar{\gamma}_{p}\right)=S_{a} R_{\gamma}\left(\bar{\gamma}_{p}\right)$

where $\bar{\gamma}_{p}$ is the effective plastic strain. The initial value of $S$ equals $S_{a}$ since the value of $R_{\gamma}$ is normalized at 1 and decreases towards 0 with increasing plastic strain. The Carreau-Yassuda function describes the softening characteristic:

$R_{\gamma}\left(\bar{\gamma}_{p}\right)=\frac{\left(1+\left(r_{0} \exp \left(\bar{\gamma}_{p}\right)\right)^{r_{1}}\right)^{\left(r_{2}-1\right) / r_{1}}}{\left(1+r_{0}{ }^{r_{1}}\right)^{\left(r_{2}-1\right) / r_{1}}}$

where $r_{0}, r_{1}$ and $r_{2}$ are fitting parameters. The material parameters for PET have been determined in a previous study [20] and are given in Table 1.

\section{Numerical model}

A 3D FEM model of the peel experiment is made. The model geometry and boundary conditions are shown in Fig. 10. The model consists of a polymer coating layer, a cohesive zone layer and a substrate. The steel is modelled as an isotropic linear elastic solid with a Young's modulus of $210 \mathrm{GPa}$ and a Poisson's ratio of 0.3. The interface has a initial zero thickness and is modelled with $3 \mathrm{D}$ cohesive zones and the presently proposed LDF. The constitutive model of the coating layer was outlined in Sect. 6 . The coating and substrate are meshed with eight-node hexahedral element with trilinear interpolation functions. Due to symmetry conditions the model only encompasses a quarter of the specimen, which is properly incorporated in the boundary conditions applied. The model is loaded by a displacement $u_{x}$ on one end of the polymer coating, as shown in Fig. 10.

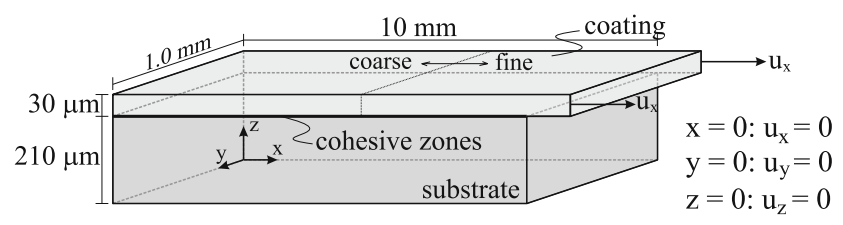

Fig. 10 The 3D peel model with boundary conditions
A mesh convergence study is performed to find the optimum element discretization, which is a compromise between the accuracy and required computational time. The mesh is divided into a coarse and a fine region, as depicted in Fig. 10. Only the part of the coating that delaminates (and thus deforms) needs to have a fine mesh, whereas the bulk coating can be handled with a relative coarse mesh. In this section the discretization of the fine coating part is further scrutinized.

The number of elements in $x, y$ and $z$ direction are varied and responses of the models are compared by considering the quasi-static peel-force, where a fine mesh should provide the best accuracy. Therefore, the peel-force $F$ is normalized by the peel-force $F_{r}$ calculated by the model with the most refined mesh. Evidently, the resulting conclusions are only valid for the range of geometries and discretization studied.

In Fig. 11a the influence of the amount of elements in $y$-direction (width) of the model is shown for two model widths. With four elements in $y$-direction the relative error is $0.5 \%$, which is considered as acceptable. The two models with different widths yield almost identical results. This might indicate that the width of the model has little influence on its response and that the geometry can be reduced to plane-strain. This is investigated in Sect.9.

The amount of elements in $z$-direction have a more pronounced influence on the peel force, up to $2 \%$ when only a single element over the thickness is used (see Fig. 11b). An error smaller than $0.5 \%$ is achieved with at least nine elements over the coating thickness.

The influence of the element size in the $x$-direction is shown in Fig. 11c, where it is also clear that the characteristic length, $\delta$, does not have a pronounced influence on the convergence. An element length of $l_{e}=2 \mu \mathrm{m}$ leads to a maximum error of $0.5 \%$.

The amount of elements in a model approximately equals: $n_{\text {elem }}=\left(1+n_{z}\right) n_{x} n_{y}$. For the number of degree-of-freedoms (DOFs) an empirical expression is used: $n_{\mathrm{DOF}}=4 n_{\text {elem }}$. The computational time for a single load step is related to $n_{\text {DOF }}$ as: $t_{\text {inc }}=\left(n_{\text {DOF }}\right)^{n}$, where $n=1.34$ (see Fig. 11d).

Simulations with different load step sizes have been carried out and it was found that the relative error remains well below $1 \%$ for all step sizes. However, with increasing step sizes, not surprisingly, convergence may be lost. The largest step size that resulted in a stable simulation was $100 \mathrm{~nm} / \mathrm{inc}$. It was found that this step size is dictated by the stability of the PET constitutive model.

It may be worth verifying whether the steps are not too large compared to the characteristic length of the cohesive zone $(\delta)$. Theoretically, a cohesive zone should dissipate the interfacial energy (per unit area) $\phi$, which is equal to the area under the curve in Fig. 6. If, however, the incremental steps are too large, the numerical integration of the tractionseparation law may yield an inaccurate dissipated energy. On 
Fig. 11 The normalized quasi-static peel forces as a function of the number of elements in $y$-direction (a), $z$-direction (b) or element length (c). The influence of the number of DOF on the CPU time of a single increment $(\mathbf{d})$
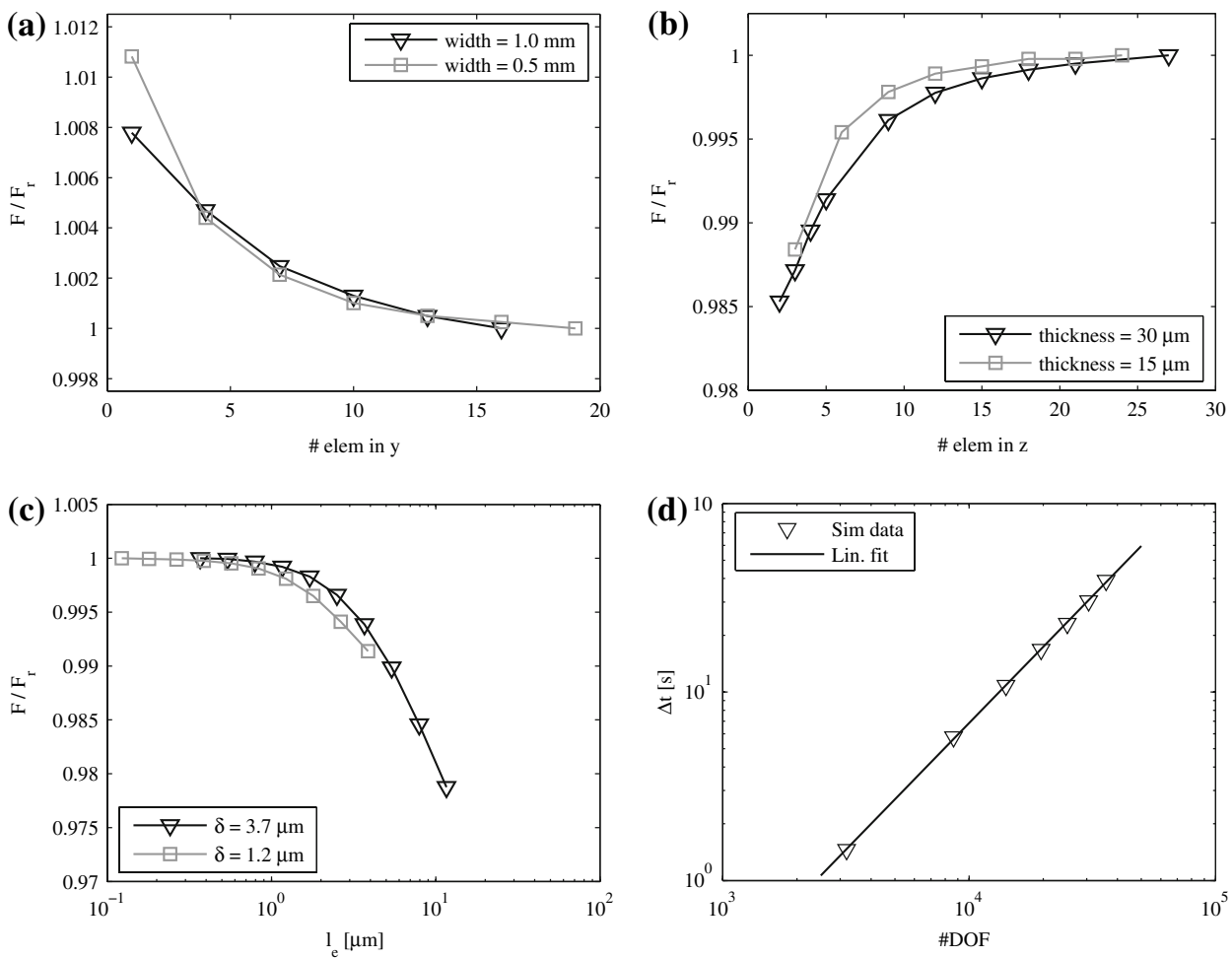

this basis, a simple analysis is performed to estimate the error as a function of the incremental opening of a cohesive zone. From this analysis it follows that the incremental cohesive zone opening should be smaller than $\frac{1}{3} \delta$ in order to dissipate more than $99 \%$ of $\phi$.

Since the displacement is controlled at the end of the polymer coating and the coating stretches at least $50 \%$, the maximum incremental step size increases to $\frac{1}{2} \delta$. For a stable simulation the step size may not be larger than $100 \mathrm{~nm} / \mathrm{inc}$. So, as long as $\delta>200 \mathrm{~nm}$, this maximum load step size can be retained.

\section{Application}

A 3D peel-off model is made with ten elements in $y$-direction and with three elements in $z$-direction. The elements have a length of $2 \mu \mathrm{m}$ in the $x$-direction. The load step size is $0.1 \mu \mathrm{m} /$ inc. Numerical results are here compared qualitatively with the experiments. These results will be used in future work to identify the interfacial parameters also quantitatively. Here the interfacial parameters are taken as $\phi=$ $200 \mathrm{~J} \mathrm{~m}^{-2}$ and $T_{\max }=20 \mathrm{MPa}$, which are in a realistic range.

In Fig. 12 an image is shown of the peel specimen. The deformed geometry can be compared with the results of the simulations, shown in Fig. 13 and a good qualitative agreement is found. The three types of unloading behaviour of the

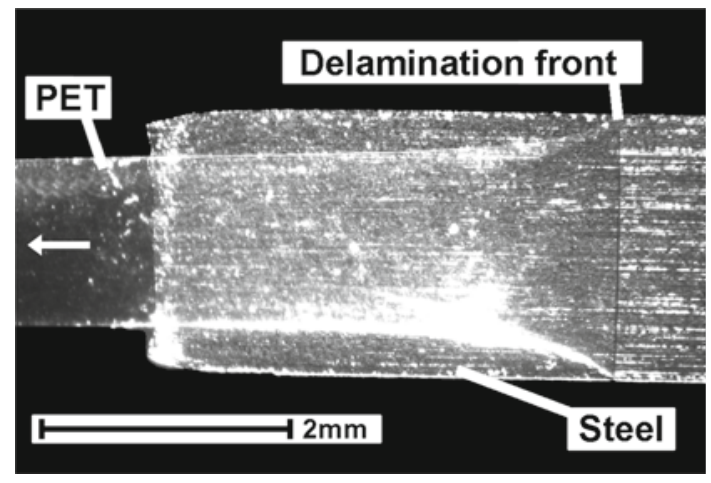

Fig. 12 Optical microscope image of the tensile specimen during a peel experiment after $3.7 \mathrm{~mm}$ of delamination

cohesive zones (see Fig. 6) do not have a noticeable influence on the simulation results because of the continuous tensile loading conditions of the structure.

\section{Plane-strain and 3D}

Three-dimensional simulations are computational expensive, so it is worthwhile to investigate the possibility to use a model with a 2D plane-strain geometry instead. In Fig. 14 the 3D and 2D models are compared by plotting their force-displacement curves. Clearly, the plane-strain model overestimates the quasi-static peel force by $4 \%$. This overestimation is 


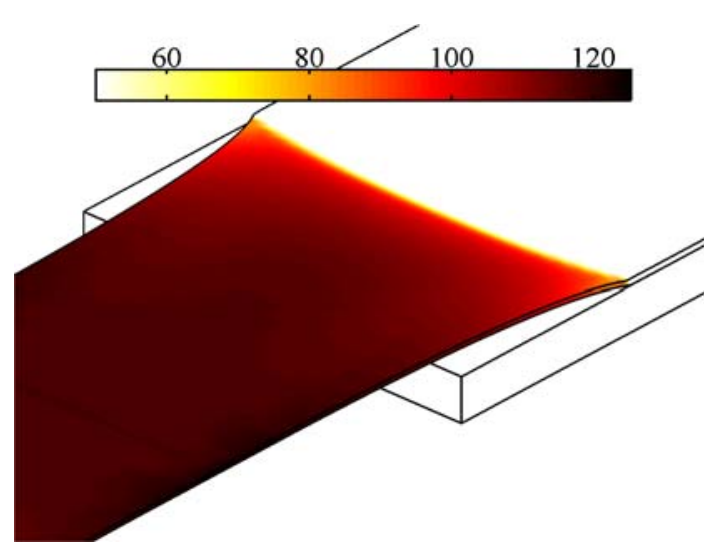

Fig. 13 Model predictions of the deformed geometry and the equivalent Von Mises stress after a delamination of $1.0 \mathrm{~mm}$. Element edges and cohesive zones are made invisible

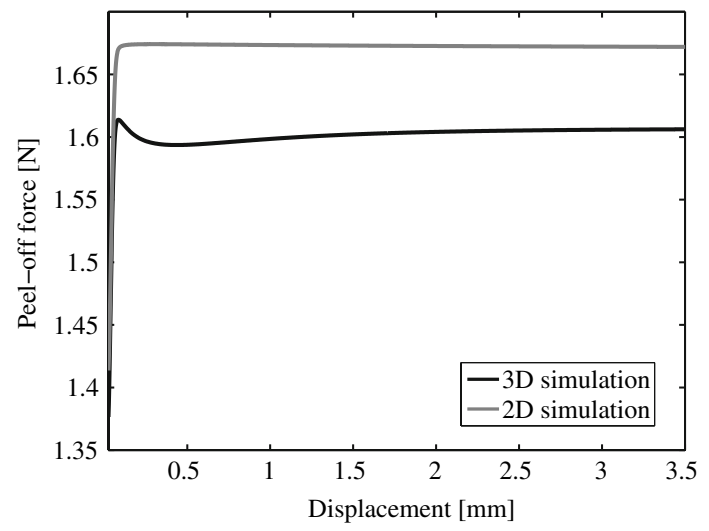

Fig. 14 The peel force as a function of the displacement as calculated by a $3 \mathrm{D}$ and a plane-strain model

caused by the plane-strain condition, which prevents deformations in $y$-direction. The advantage of the $2 \mathrm{D}$ model with respect to the 3D model is the significant reduction in calculation time. Depending on the width of the model and thus the element discretization in the width direction, the calculation time is typically reduced by a factor $15-50$. This makes a plane-strain model an attractive alternative to perform qualitative parameter studies and useful in the first steps towards an iterative fitting procedure on quantitative experimental results.

\section{Conclusion}

It is shown that delamination in polymer coated steels typically takes place through a process of fibrillation. This mechanism involves large displacements and deformations at the interface and in the surrounding bulk materials. Cohesive zone models are a convenient tool to describe this failure mechanism. However, classical cohesive zone models cannot cope with large displacements and deformations in a consis- tent way because these models use a local coordinate basis to decompose the opening displacement. A LDF is proposed to overcome the shortcomings of the classical SDF. In the LDF a global basis is used and only one relation between the traction and the opening displacement is defined. As a consequence there is only a single mode-independent work-of-separation, which is a valid assumption regarding the presence of fibrils at the interface. A more general LDF cohesive zone model with a mode-dependent work-of-separation may be more relevant for other material systems.

A 3D cohesive zone element with the LDF is developed and implemented in a finite element solution framework. The detection of contact in a cohesive zone element with the LDF is no longer straightforward and has been solved by defining a normal vector with respect to the cohesive zone element mid-plane.

The simulation of a peel experiment with the use of the presented 3D model illustrates the added value of the developed 3D cohesive zone element with the LDF. Good qualitative agreement is found between the model predictions and experimental observations of a peel-off test. Future work consists in quantitative determination of the interface parameters on the basis of detailed experimental measurements.

Acknowledgments This research was carried out under project number MC2.03149 in the framework of the Strategic Research Programme of the Netherlands Institute for Metals Research in The Netherlands (www.nimr.nl).

\section{References}

1. Abdul-Baqi A, Van der Giessen E (2001) Indentation-induced interface delamination of a strong film on a ductile substrate. Thin Film Solids 381:143-154

2. Baaijens FPT (1991) Calculation of residual stresses in injectionmolded products. Rheol Acta 30:284-299

3. Boelen B, Den Hartog H, Van der Weijde H (2004) Product performance of polymer coated packaging steel, study of the mechanism of defect growth in cans. Prog Org Coat 50:40-46

4. Chandra N, Li H, Shet C, Ghonem H (2002) Some issues in the application of cohesive zone models for metal-ceramic interfaces. Int J Solids Struct 39:2827-2855

5. Chvedov D, Jones R (2004) Frictional behavior of rolled surfaces coated with polymer films. Surface Coat Technol 188-189:544549

6. Cornec A, Scheider I, Schwalbe KH (2003) On the practical application of the cohesive model. Eng Frac Mech 70:1963-1987

7. De Borst R (2003) Numerical aspects of cohesive-zone models. Eng Frac Mech 70:1743-1757

8. Eyring H (1936) Viscosity, plasticity, and diffusion as examples of absolute reaction rates. J Chem Phys 4:283-295

9. Govaert LE, Timmermans PHM, Brekelmans WAM (2000) The influence of instrinsic strain softening on strain localization in polycarbonate: modeling and experimental validation. J Eng Mat Tech 122:177-185

10. Graziano F (2000) Coil and sheet coating. Metal Finish 98:175176 
11. Haward R, Thackray G (1968) The use of a mathematical model to describe isothermal stress-strain curves in glassy polymers. Proc R Soc Lond A 302:453-472

12. Klompen ETJ, Engels TAP, Govaert LE, Meijer HEH (2005) Modeling of the postyield response of glassy polymers: inlfuence of thermomechanical history. Macromolecules 38:6997-7008

13. Leonov AI (1976) Nonequilibrium thermodynamics and rheology of viscoelastic polymer media. Rheol Acta 15:85-98

14. Qiu Y, Crisfield MA, Alfano G (2001) An interface element formulation for the simulation of delamination with buckling. Eng Frac Mech 68:1755-1776

15. Roychowdhury S, Arun Roy Y, Dodds RH (2002) Ductile tearing in thin aluminium panels: experiments and analyses using large-displacement, 3-D surface cohesive elements. Eng Frac Mech 69:983-1002

16. Tervoort TA, Govaert LE (2000) Strain-hardening behavior of polycarbonate in the glassy state. J Rheol 44:1263-1277
17. Tvergaard V (1990) Effect of fibre debonding in a whiskerreinforced metal. Mat Sci Eng A 125:203-213

18. Van den Bosch MJ, Schreurs PJG, Geers MGD (2006) An improved description of the exponential $\mathrm{Xu}$ and Needleman cohesive zone law for mixed-mode decohesion. Eng Frac Mech 73:1220-1234

19. Van den Bosch MJ, Schreurs PJG, Geers MGD (2007) A cohesive zone model with a large displacement formulation accounting for interfacial fibrilation. Eur J Mech 26:1-19

20. Van der Aa HCE, Van der Aa MAH, Schreurs PJG, Baaijens FTP, Van Veenen WJ (2000) An experimental and numerical study of the wall ironing process of polymer coated sheet metal. Mech Mat 32:423-443

21. Xu XP, Needleman A (1993) Void nucleation by inclusions debonding in a crystal matrix. Mod Sim Mat Sci Eng 1:111-132 